\title{
Glycogen phosphorylase BB levels are associated with haemodynamic parameters in cirrhotic patients undergoing transjugular intrahepatic portosystemic shunt insertion
}

\author{
Martina Vasatovaa ${ }^{a}$, Radek Pudilib, Tomas Buchlerc, Iva Karesovaa , Vaclav Safka ${ }^{d}$, Tomas Fejfard, Petr Hulek ${ }^{d}$
}

\begin{abstract}
Background. Transjugular intrahepatic portosystemic shunts (TIPS) have become a widely accepted tool in the treatment of patients with symptomatic portal hypertension. The aim of our study was to assess glycogen phosphorylase BB (GPBB) concentration in relation to echocardiographic and haemodynamic parameters in patients before and after TIPS insertion.

Methods. The study population consisted of 55 patients ( 38 men and 17 women, age $55.6 \pm 8.9$ years, range $37-74$ years) with liver cirrhosis treated with transjugular portosystemic shunting. GPBB, echocardiographic, and haemodynamic parameters were measured before TIPS insertion and $24 \mathrm{~h}$ after the procedure. GPBB concentrations were assessed using the Cardiac Array for Evidence Investigator protein biochip. Correlation between parameters was assessed using the Spearman's coefficient.

Results. Serum post-procedural GPBB concentrations were increased in comparison with baseline $(5.58 \mathrm{vs} .2 .67 \mu \mathrm{g} / \mathrm{L}$, $P<0.001)$. GPBB concentration after TIPS significantly correlated with baseline systemic vascular resistence $(r=0.330$; $P=0.017)$ and cardiac index $(r=0.313 ; P=0.025)$.
\end{abstract}

Conclusion. GPBB concentration measurement may be a useful tool for monitoring myocardial ischemia during a TIPS procedure.

Key words: glycogen phosphorylase BB, cardiac marker, cirrhosis, biochip, portal hypertension, TIPS

Received: October 22, 2013; Accepted: April 7, 2014; Available online: April 29, 2014

http://dx.doi.org/10.5507/bp.2014.020

aInstitute of Clinical Biochemistry and Diagnostics, University Hospital in Hradec Kralove, Czech Republic

${ }^{b} 7^{\text {st }}$ Department of Internal Medicine - Cardioangiology, University Hospital in Hradec Kralove and Faculty of Medicine in Hradec Kralove, Charles University in Prague

'Department of Oncology, $1{ }^{\text {st }}$ Faculty of Medicine, Charles University in Prague and Thomayer University Hospital, Prague

${ }^{d} 2^{\text {nd }}$ Department of Internal Medicine-Gastroenterology, University Hospital in Hradec Kralove and Faculty of Medicine in Hradec Kralove, Charles University in Prague

Corresponding author: Martina Vasatova, e-mail: martina.vasatova@fnhk.cz

\section{INTRODUCTION}

Portal hypertension is the main cause for the development of esophago-gastric varices, ascites and hepatic nephropathy in liver cirrhosis. Transjugular intrahepatic portosystemic shunts (TIPS) have become widely accepted for the treatment of patients with symptomatic portal hypertension. TIPS decreases the portal pressure by redirecting portal blood volume into the systemic venous circulation. However, subsequent marked increase in ventricular preload can precipitate cardiac dysfunction ${ }^{1}$. Several studies have reported changes in echocardiography and invasive haemodynamic monitoring parameters after TIPS insertion. Huonker et al. observed an increase in the left atrial diameter, left ventricular end diastolic volume, pulmonary capillary wedge pressure, right atrial pressure, cardiac output, and total pulmonary resistance after TIPS procedure ${ }^{2}$. These changes reflect diastolic dysfunction occurring after TIPS. Schwartz et al. reported that congestive heart failure developed in $13 \%$ patients after TIPS insertion ${ }^{3}$. Bernal et al. and Henriksen et al. found high level of NT-proBNP levels in cirrhotic patients possibly due to diastolic dysfunction and central volume expansion ${ }^{4,5}$. On the other hand, there are only several studies measuring cardiac necrosis markers in relation to TIPS procedure ${ }^{6,7}$. In the last decade, the armamentarium of cardiac markers has expanded to include several molecules that are potentially useful for the diagnosis of cardiac injury and are awaiting validation for routine clinical applications. These novel analytes include glycogen phosphorylase BB isoenzyme (GPBB) as marker of myocardial ischemia and necrosis ${ }^{8}$.

The aim of our study was to measure GPBB concentration to assess their relationship to echocardiographic and haemodynamic parameters in patients before TIPS insertion - mainly increased heart rate, cardiac output (cardiac index), and reduced systemic vascular resistance.

\section{MATERIALS AND METHODS}

\section{Study population}

Patients with liver cirrhosis regardless of aetiology requiring the insertion of TIPS were enrolled in the study. 
Table 1. Shows patient demographic characteristics.

\begin{tabular}{|c|c|c|}
\hline \multicolumn{3}{|l|}{ Sex: } \\
\hline & Men n (\%) & $38(69)$ \\
\hline & Women n (\%) & $17(31)$ \\
\hline \multicolumn{3}{|l|}{ Age: } \\
\hline & All patients $(x \pm S D)$ & $55.6 \pm 8.9$ \\
\hline & Women $(\mathrm{x} \pm \mathrm{SD})$ & $55.1 \pm 9.4$ \\
\hline & $\operatorname{Men}(x \pm S D)$ & $55.8 \pm 8.7$ \\
\hline \multicolumn{3}{|c|}{ Aetiology: } \\
\hline & Alcohol n (\%) & $36(65)$ \\
\hline & Viral n (\%) & $7(13)$ \\
\hline & NASH n (\%) & $6(11)$ \\
\hline & Autoimmune n (\%) & $2(4)$ \\
\hline & Unknown n (\%) & $4(7)$ \\
\hline \multicolumn{3}{|c|}{ Child-Pugh score: } \\
\hline & points $(\mathrm{x} \pm \mathrm{SD})$ & $8.7 \pm 1.5$ \\
\hline \multicolumn{3}{|c|}{ Child classification: } \\
\hline & A n $(\%)$ & $7(13)$ \\
\hline & B n $(\%)$ & $32(58)$ \\
\hline & C n $(\%)$ & $16(29)$ \\
\hline
\end{tabular}

SD - standard diviation, NASH - non-alcoholic steatohepatitis ${ }^{6}$.

Patients were required to be in a stable condition with no gastrointestinal bleeding during the 15 days preceding the TIPS insertion. Medication affecting haemodynamics, such as $\beta$-blockers and vasodilators, was stopped at least 10 days before TIPS. Diuretics were kept constant during the week before the TIPS insertion. Patency of the shunt was evaluated using the Doppler ultrasound during regular follow-up. The study protocol was approved by the local Ethics Committee. Informed consent was obtained from each patient.

\section{Haemodynamic monitoring}

All patients were monitored using Swan-Ganz pulmonary catheter inserted in the pulmonary circulation before the TIPS procedure. The haemodynamic parameters including heart rate, blood pressure, cardiac output (CO), cardiac index (CI), systemic vascular resistance (SVR), pulmonary vascular resistance (PVR), and pulmonary capillary wedge pressure (PCWP) were measured before the procedure and $24 \mathrm{~h}$ after the TIPS insertion.

Echocardiography was performed in agreement with the American Society of Echocardiography standards evaluating the following parameters: left ventricular enddiastolic (LVEDD) and end-systolic (LVESD) diameters, left atrium diameter (LA), right ventricle diameter (RV), end-diastolic septal and posterior wall thickness, inferior vena cava diameter (IVC), LV ejection fraction, peak early filling velocity (E), peak late atrial filling velocity (A), E/A ratio, deceleration time of E velocity (DT-E) and isovolumic relaxation time (IVRT).
Table 2. Shows haemodynamic and echocardiographic parameters.

\begin{tabular}{|c|c|c|}
\hline & Before TIPS & $24 \mathrm{~h}$ after TIPS \\
\hline \multicolumn{3}{|c|}{ Echocardiographic parameters: } \\
\hline $\mathrm{LA}(\mathrm{mm})$ & $39.5(36.2-43.4)$ & $38.5(34.42 .2)$ \\
\hline $\mathrm{RV}(\mathrm{mm})$ & $27(25-29)$ & $28(26-30)$ \\
\hline $\operatorname{LVESD}(\mathrm{mm})$ & $31.5(30.1-34.3)$ & $30(27.2-34.8)$ \\
\hline LVEDD (mm) & $47.0(44.7-51.2)$ & $46.0(44.6-48.7)$ \\
\hline IVC (mm) & $17.0(15.5-19.0)$ & $17.0(15.1-19.4)$ \\
\hline PCWP (mmHg) & $9.5 \pm 1.9$ & $10.7 \pm 3.6 *$ \\
\hline $\mathrm{CO}(\mathrm{L} / \mathrm{min})$ & $7.2 \pm 1.7$ & $9.5 \pm 1.9 * * *$ \\
\hline $\mathrm{CI}\left(\mathrm{L} / \mathrm{min} / \mathrm{m}^{2}\right)$ & $4.3 \pm 2.3$ & $5.2 \pm 1.1$ * \\
\hline SVR (dyn.sec/cm $\left.{ }^{5}\right)$ & $908 \pm 277$ & $631 \pm 133 * * *$ \\
\hline PVR (dyn.sec/ $\mathrm{cm}^{5}$ ) & $89.3 \pm 51.6$ & $82.3 \pm 43.3$ \\
\hline \multicolumn{3}{|c|}{ Hemodynamic parameters: } \\
\hline $\mathrm{E}(\mathrm{cm} / \mathrm{s})$ & $75.5(60.5-87.3)$ & $88.0(74.3-109.7)$ ** \\
\hline $\mathrm{A}(\mathrm{cm} / \mathrm{s})$ & $85.1(76.2-99.5)$ & $91.2(81.5-104.5) *$ \\
\hline $\mathrm{E} / \mathrm{A}$ & 0.887192 & $0.964912 *$ \\
\hline DT-E (ms) & $210(165-265)$ & $175(134-230)$ * \\
\hline IVRT (ms) & $70.2(60.3-80.4)$ & $75.0(62.5-89.5) *$ \\
\hline
\end{tabular}

median (interquartile range) or average $\pm \mathrm{SD},{ }^{*} P<0.05, * * P<0.01$, $* * * P<0.001, \mathrm{CO}$ - cardiac output, CI - cardiac index, SVR - systemic vascular resistance, PVR-pulmonary vascular resistance, PCWP - pulmonary capillary wedge pressure, LVEDD - left ventricular end-diastolic diameter, LVESD - left ventricular end-systolic diameter, LA- left atrium diameter, RV - right ventricule diameter, EF- left ventricular ejection fraction, E - peak early filling velocity, A - peak late atrial filling velocity, DT-E deceleration time of E velocity, IVRT- isovolumic relaxation time, IVC - inferior vena cava diameter ${ }^{6}$.

\section{Measurement of GPBB}

GPBB concentrations were measured in peripheral venous sample before the procedure and $24 \mathrm{~h}$ after the procedure. Blood samples were obtained by venepuncture, immediately shipped to the laboratory and centrifuged $(2500 \mathrm{~g}, 10 \mathrm{~min}$ ) within one hour of collection. Serum samples were stored at $-70{ }^{\circ} \mathrm{C}$ until analysis (within nine months).

GPBB concentrations were measured using the Evidence Investigator protein biochip system (Randox Laboratories, UK). Simultaneous quantitative detection of multiple analytes based on sandwich chemiluminescent immunoassay was carried out from a single patient sample. The core technology consists of a solid plate containing discrete test regions with immobilised antibodies specific to different cardiac markers. Increased levels of cardiac markers in a specimen lead to increased binding of antibody labelled with horseradish peroxidase and thus to an increase in the luminescent signal emitted. The light signals generated from each of the test regions on the bio- 


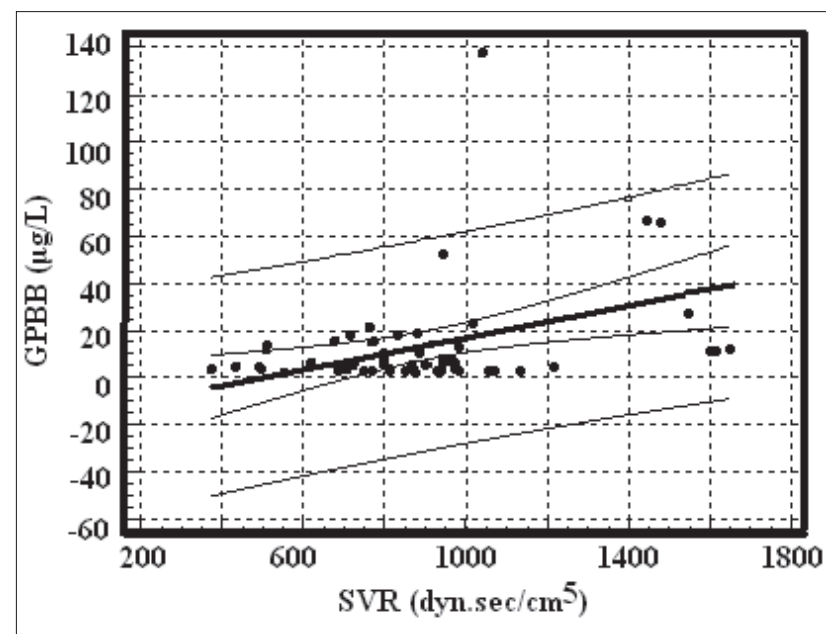

Fig. 1. Association of serum GPBB measured $24 \mathrm{~h}$ after TIPS with systemic vascular resistence (SVR) before TIPS.

chip are simultaneously detected using a charge coupled device (CCD) camera.

The analytical sensitivity of the GPBB assay was $0.65 \mu \mathrm{g} / \mathrm{L}$. We have used the cut-off value $7.3 \mu \mathrm{g} / \mathrm{L}$ as recommended by the manufacturer. The interassay coefficient of variability $(n=10)$ was in accordance with data provided by the manufacturer in the range of $\mathrm{CV}=2.0$ $9.8 \%(8.4 \mu \mathrm{g} / \mathrm{L}-6.6 \%, 68.1 \mu \mathrm{g} / \mathrm{L}-7.9 \%)$. Internal quality control measurements were carried out using samples provided by the kit manufacturer.

\section{Statistical analysis}

Normality was tested using the Kolmogorov-Smirnov test. Normally distributed data are reported as means \pm standard deviation, while non-normal data as median and interquartile range. Statistical comparisons carried out using paired Wilcoxon test for data with non-normal distribution and using paired t-tests for data with normal distribution. Differences with $P<0.05$ were considered statistically significant. Degree of association between continuous data was calculated using the Spearman's correlation coefficient.

\section{RESULTS}

The study population consisted of 55 consecutive pa-

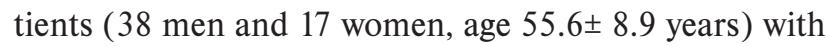
liver cirrhosis treated with elective transjugular portosystemic shunting. The cause of liver disease were alcohol consumption in $36(65 \%)$ patients, viral hepatitis 7 (13\%) patients, non-alcoholic steatohepatitis in $6(11 \%)$ patients, autoimmune hepatitis in $2(4 \%)$ patients, and cryptogenic in $4(7 \%)$ patients. The demographic characteristics are shown in Table 1.

Haemodynamic and echocardiographic parameters in patients before and $24 \mathrm{~h}$ after TIPS are shown in Table 2. Before the procedure, serum GPBB concentrations were increased over the cut-off value $7.3 \mu \mathrm{g} / \mathrm{L}$ in $12.0 \%$ of patients. Statistically significant increases in serum levels

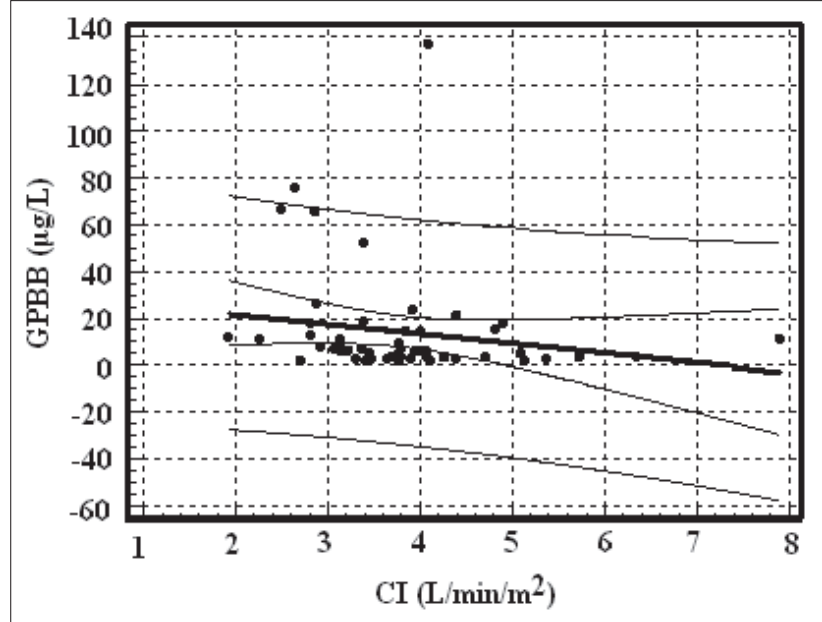

Fig. 2. Association of serum GPBB measured $24 \mathrm{~h}$ after TIPS with cardiac index $(\mathrm{CI})$ before TIPS.

of GPBB in comparison with pre-procedural levels was detected in samples obtained $24 \mathrm{~h}$ after TIPS (2.67 (2.00$5.33) \mu \mathrm{g} / \mathrm{L}$ vs. 5.58 (2.41-14.18) $\mu \mathrm{g} / \mathrm{L}, P<0.001)$. In $40 \%$ of patients, the level was above the cut-off value.

GPBB is a sensitive marker of saccharide metabolism in cardiomyocytes and this alteration was probably linked to changes in myocardial metabolism without myocardial necrosis during the procedure. We have found an association between GPBB measured $24 \mathrm{~h}$ after TIPS insertion with systemic vascular resistence (SVR) (Fig. 1) and cardiac index (CI) (Fig. 2) assessed before the procedure. The Spearman's coefficient values were as follows: GPBB vs. SVR: $\mathrm{r}=0.330(P=0.017)$; GPBB vs. $\mathrm{CI}$ : $\mathrm{r}=0.313(P=0.025)$.

\section{DISCUSSION}

The transjugular intrahepatic portosystemic shunt is widely used to treat portal hypertension-related complications. TIPS may impair cardiac function and haemodynamics. In particular, it rapidly shifts a large volume of blood from the splanchnic area to the heart, thus increasing the cardiac preload. Liver cirrhosis is frequently associated with cardiac dysfunction (cirrhotic cardiomyopathy) and circulation abnormalities.

To our knowledge, this is the first report on GPBB association with haemodynamic parameters in patients undergoing TIPS insertion. According to a recent definition of myocardial infarction, cardiospecific troponins are recommended for the detection of myocardial injury as cardiac markers of the first choice ${ }^{9,10}$. However, we found no differences in concentrations of common cardiac markers ${ }^{7}$. In contrast, a significant increase in the serum GPBB concentration occurred during TIPS insertion. GPBB is a glycolytic enzyme that plays an essential role in the regulation of carbohydrate metabolism. It catalyzes the first step of glycogenolysis, where glycogen is converted to glucose 1-phosphate by phosphorolysis in the presence of inorganic phosphate, and through 
mobilization of glycogen, it is primarily associated with provision of an emergency glucose supply during periods of hypoxia and hypoglycemia. Under normal conditions, GPBB is structurally bound to macromolecular complex in the sarcoplasmatic reticulum. With the onset of tissue ischemia, GPBB is converted to a free soluble form and diffuses out of the cell if the membrane permeability is simultaneously increased. GPBB usually peaks $6-20 \mathrm{~h}$ after myocardial infarction onset and returned within the reference interval within 1-2 days ${ }^{11}$. Diagnostic sensitivity and specificity GPBB for myocardial infarction diagnosis are 0.81 and 0.93 , respectively. The area under ROC curve is $0.85-0.87\left(\right.$ ref. $\left.{ }^{12}\right)$.

GPBB is a promising enzyme for the laboratory detection of early ischemic myocardial damage ${ }^{8}$. The GPBB concentration increase during TIPS may be linked to changes in myocardial metabolism without myocardial necrosis during the procedure.

We found an association between the serum GPBB levels measured $24 \mathrm{~h}$ after the TIPS insertion and hemodynamic parameters including systemic vascular resistance and cardiac index assessed before the procedure. Cardiac output and cardiac index are dependent on the left ventricle function. After the TIPS procedure, both cardiac output and cardiac index increase because of the decrease in systemic vascular resistance. Therefore, the positive association of GPBB with systemic vascular resistance and negative association with cardiac index probably indicates decreased cardiac contractile reserve. This observation can be helpful for identifying patients with increased cardiovascular risk. Positivity of GPBB in patients with negativity of other cardiomarkers suggests that GPBB could be a more sensitive marker for detection of acute cardiac injury. Horacek et al. reported increased GPBB associated with cardiotoxicity caused by anticancer therapy ${ }^{13}$.

However, hepatic encephalopathy is also a common complication following the insertion of TIPS and GPBB is also the predominant isoenzyme in the human brain. Much lower GPBB concentrations have been reported in leukocytes, platelets, spleen, kidney, bladder, testis, digestive tract and aorta. On the other hand, the liver contains predominantly the GPLL isoenzyme GPLL.

\section{CONCLUSIONS}

Our study was a pilot non-randomized study with a relatively small number of cirrhotic patients undergoing a TIPS procedure. Our results show that 1) serum postprocedural GPBB concentrations are increased in comparison with baseline; 2) serum post-procedural GPBB concentrations are associated with hemodynamic parameters including systemic vascular resistance and cardiac index. The GPBB may be a useful tool for monitoring myocardial ischemia occurring during the TIPS procedure.

\section{ABBREVIATIONS}

A, Peak late atrial filling velocity; CI, Cardiac index; CO, Cardiac output; DT-E, Deceleration time of E velocity; E, Peak early filling velocity; EF, Left ventricular ejection fraction; GPBB, Glycogenphosphorylase BB isoenzyme; IVC, Inferior vena cava diameter; IVRT, Isovolumic relaxation time; LA, Left atrium diameter; LVEDD, Left ventricular end-diastolic diameter; LVESD, Left ventricular end-systolic diameter; PCWP, Pulmonary capillary wedge pressure; PVR, Pulmonary vascular resistance; RV, Right ventricule diameter; SVR, Systemic vascular resistance; TIPS, transjugular intrahepatic portosystemic shunt.

\section{ACKNOWLEDGEMENT}

The study was supported by MH CZ - DRO (UHHK, 00179906) and the programme PRVOUK P37/3.

Author contributions: MV, IK: biochemical and statistical analysis; RP: cardiology, project leader; VS, TF, PH: hepatology, TIPS, selection of patients; TB: manuscript revision, editing and translation.

Conflict of interest statement: The authors declare that there are no conflicts of interest regarding the publication of this article.

\section{REFERENCES}

1. Lee RF, Glenn TK, Lee SS. Cardiac dysfunction in cirrhosis. Best Pract Res Clin Gastroenterol 2007;21:25-40.

2. Huonker M, Schumacher YO, Ochs A, Sorichter S, Keul J, Rössle M. Cardiac function and haemodynamics in alcoholic cirrhosis and effects of the transjugular intrahepatic portosystemic stent shunt. Gut 1999;44:743-8.

3. Schwartz JM, Beymer C, Althaus SJ, Larson AM, Zaman A, Glickerman DJ, Kowdley KV. Cardiopulmonary consequences of transjugular intrahepatic portosystemic shunts: role of increased pulmonary artery pressure. J Clin Gastroenterol 2004;38:590-4.

4. Bernal V, Pascal I, Esquivias P, García-Gil A, Fernández C, Mateo JM, González M, Simón MA. Cardiac hemodynamic profiles and proB-type natriuretic peptide in cirrhotic patients undergoing liver transplantaion. Transplant P 2009; 41:985-6.

5. Henriksen JH, Gøtze JP, Fuglsang S, Christensen E, Bendtsen F, Møller S. Increased circulating pro-brain natriuretic peptide (proBNP) and brain natriuretic peptide (BNP) in patients with cirrhosis: relation to cardiovascular dysfunction and severity of disease. Gut 2003;52:1511-7.

6. Pudil R, Praus R, Hůlek P, Safka V, Fejfar T, Vasatova M, Vojacek J. Hemodynamic and echocardiographic changes in patients with liver cirrhosis treated with transjugular intrahepatic portosystemic shunt. Interv Akut Kardiol 2013;12:8-11.

7. Vasatova M, Pudil R, Safka V, Fejfar T, Buchler T, Hulek P, Palicka V. Elevated cardiac markers are associated with higher mortality in patients after transjugular intrahepatic portosystemic shunt insertion. Ann Clin Biochem 2013;50:122-6.

8. Mair J. Glycogen phosphorylase isoenzyme BB to diagnose ischemic myocardial damage. Clin Chim Acta 1998;272:79-86.

9. Thygesen K, Albert JS, White HD. Universal Definition of Myocardial Infarction. J Am Coll Cardiol 2007;50:2173-95.

10. K. Thygesen, J.S. Alpert, A.S. Jaffe et al. Third universal definition of myocardial infarction, Circulation 2012;126:2020-35.

11. Apple FS, Wu AHB, Mair J, Ravkilde J, Panteghini M, Tate J, Pagani F, Christenson RH, Mockel M, Danne O, Jaffe AS. Future Biomarkers 
for Detection of Ischemia and Risk Stratification in Acute Coronary Syndrome. Clin Chem 2005;51:810-24.

12. Rabitzsch G, Mair J, Lechleitner P, Noll F, Hofmann U, Krause EG, Dienstl F, Puschendorf B. Immunoenzymatic assay of human glycogen phosphorylase isoenzyme BB in diagnosis of ischemic myocardial injury. Clin Chem 1995;41:966-78.

13. Horaček JM, Vasatova M, Tichy M, Pudil R, Jebavy L, Maly J. The use of cardiac biomarkers in detection of cardiotoxicity associated with conventional and high-dose chemotherapy for acute leukemia. Exp Oncol 2010;32,97-9. 